\title{
CK Vul as a candidate eruptive stellar merging event
}

\begin{abstract}
T. Kato ${ }^{\star}$
Department of Astronomy, Kyoto University, Kyoto 606-8502, Japan

Received 25 October 2002 / Accepted 3 December 2002

Abstract. CK Vul (Nova Vul 1670) is one of the most mysterious objects among erupting stellar objects. Past studies have suggested that CK Vul is a final helium-flash object resembling V605 Aql and V4334 Sgr (Sakurai's object). The peculiar outburst light curve of CK Vul, however, had no similar counterpart among the known eruptive objects. Furthermore, the presence of hydrogen in the proposed remnant seems to contradict with the final helium-flash scenario. We propose that the peculiarities of CK Vul can be naturally understood if we consider a merging of main-sequence stars, following a new interpretation by Soker \& Tylenda (2003) which was proposed to explain the peculiar eruptive object V838 Mon. In this case, the 1670 outburst of CK Vul may be best understood as a V838 Mon-like event which occurred in our vicinity.
\end{abstract}

Key words. accretion, accretion disks - stars - novae, cataclysmic variables - stars: individual: CK Vul

\section{Introduction}

CK Vul (Nova Vul 1670) is one of the most mysterious objects among erupting stellar objects. The object reached a maximum of $m_{v}=2.6$, with at least three brightness peaks spanning for three years. The overall light curve and the multiple brightness peaks spanning for such a long period is unlike any classical nova (Payne-Gaposchkin 1957; Duerbeck 1981; Duerbeck 1987). The object was claimed to be recovered as the "oldest old nova" (Shara \& Moffat 1982) from the presence of a planetary nebula-like shell. Shara et al. (1985) further proposed, from the low absolute magnitude of the supposed quiescent counterpart, that CK Vul is a "hibernating" old nova (cf. Shara et al. 1986).

Harrison (1996), however, performed near-infrared photometry of the suggested counterpart, and yielded a spectral energy distribution (SED) indicating the presence of a dust shell. From the similarity of SED and the outburst light curve with those of V4334 Sgr (Sakurai's object), Harrison (1996) suggested that CK Vul may be related to V4334 Sgr, which is believed to be a final helium-flash object (Duerbeck et al. 1997; Kerber 1999) rather than a classical nova. Evans et al. (2002) presented further evidence for a final helium-flash object from the detection of a cavity in IRAS infrared map and the analysis of sub-mm emission.

However, it is controversial whether the unique light curve of CK Vul can be reasonably explained by the scenario of a final helium-flash object. Although both V605 Aql and V4334 Sgr had multiple optical peaks, the later peaks were always fainter than the preceding peaks. This phenomenon is natural since the optical variations are regarded as a consequence of a combination of continuous cooling and dust formation (Asplund et al. 1999). As pointed out by Shara et al. (1985), a nova-type thermonuclear runaway event should decay

\footnotetext{
* e-mail: tkato@kusastro.kyoto-u.ac.jp
}

with a dynamical time-scale $(\sim 100 \mathrm{~d})$ from a super-Eddington maximum phase, which further makes it difficult to explain the long intervals between the brightenings.

Most recently, a unique eruptive object, V838 Mon, has been discovered (Brown et al. 2002). The subsequent evolution of V838 Mon, with multiple optical peaks and very cool atmosphere (Munari et al. 2002b) has been remarkably unlike any class of erupting stellar objects. The light curve of V838 Mon in part resembles that of $\mathrm{CK}$ Vul, particularly in its second bright maximum long time after the initial eruption (Fig. 1). Although the exact mechanism of the eruption of V838 Mon has not been clarified, Soker \& Tylenda (2003) proposed that a merging of two main-sequence stars can explain the unique outburst of V838 Mon. Based on the phenomenological resemblance between CK Vul and V838 Mon, we try to explore the possibility of explaining CK Vul within the framework of Soker \& Tylenda (2003).

\section{Application to CK Vul}

According to Soker \& Tylenda (2003), the eruption of V838 Mon has been proposed to be a merging of a close main-sequence binary with $M_{1} \sim 1.5 M_{\odot}$ and $M_{2} \sim$ $0.1-0.5 M_{\odot}$. The first maximum was explained as a runaway mass-transfer when the primary (more massive) star fills the Roche-lobe. The observed luminosity of the initial maximum can be well reproduced by a reasonable parameter in a calculation of an accreting $0.2 M_{\odot}$ main-sequence star (Prialnik \& Livio 1985) and the subsequent expansion to a giant. Soker \& Tylenda (2003) proposed that the second (brighter) maximum can be explained by the accretion of the secondary onto the primary, particularly by the merging of the stellar cores, which enables a further expansion (Hjellming \& Taam 1991).

The scenario by Soker \& Tylenda (2003) thus seems to able to explain the reason why the second maximum of V838 Mon 


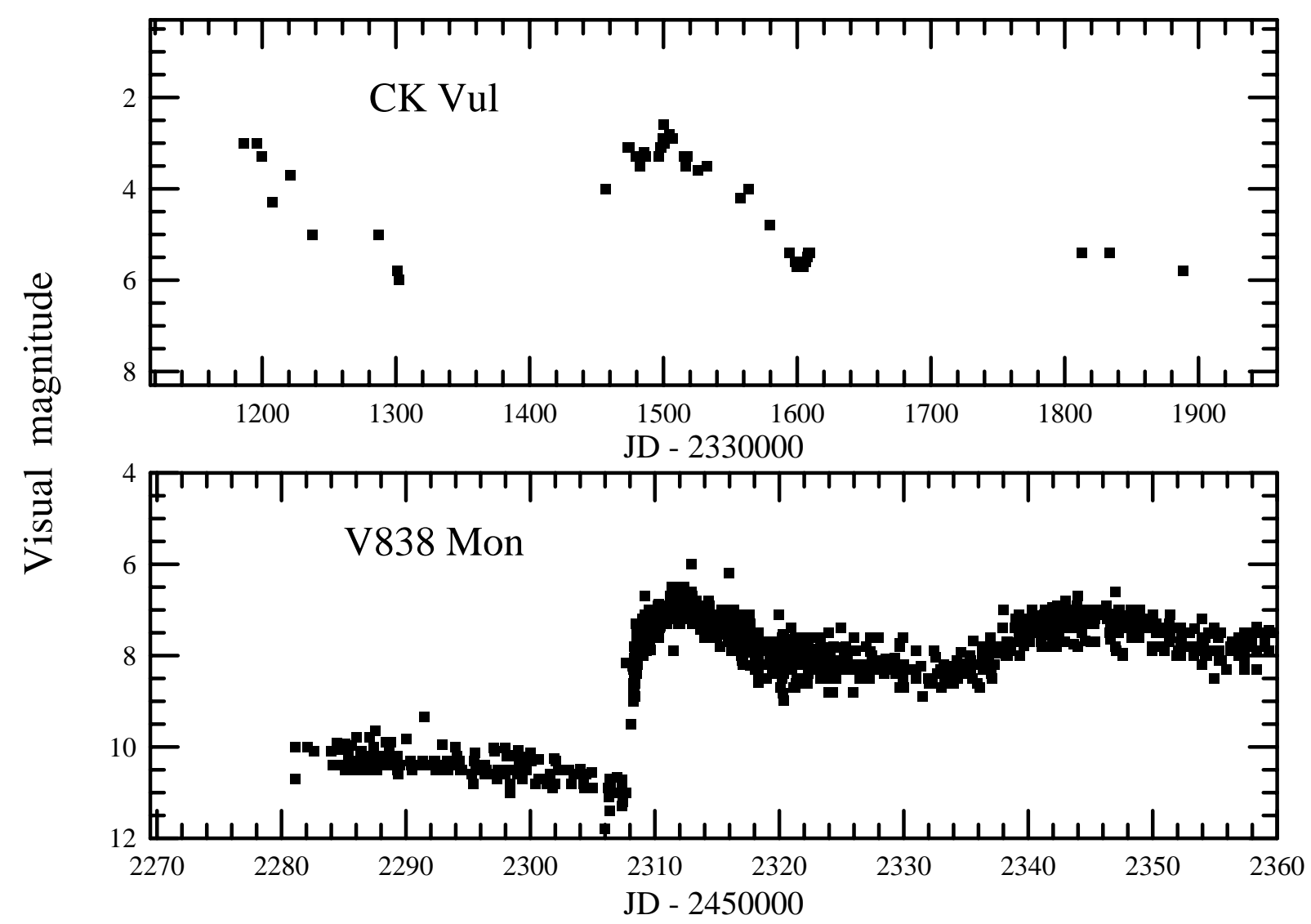

Fig. 1. Comparison of the light curves of CK Vul and V838 Mon. The data of CK Vul are from Shara et al. (1985). The data of V838 Mon (visual and $V$-band observations) are from the reports to VSNET.

was more energetic than the first maximum. In CK Vul (Shara et al. 1985), the second maximum was recorded to be at least as bright as the first (initially discovered) maximum. In Soker \& Tylenda (2003), the brightness of the first maximum depends on the parameter $\alpha$, which is a fraction of the free-fall energy employed to expand the atmosphere. In reproducing V838 Mon, Soker $\&$ Tylenda (2003) used $\alpha=0.1$, while a higher value (up to $\alpha=0.5$ ) is realistic. The time-scales between the first and second maximum can be strongly different depending on the initial condition of the binary and the degree of angular momentum loss, both of which are not still reasonably constrained.

A subtle difference between the light curves of $\mathrm{CK}$ Vul and V838 Mon is not thus problematic within the scenario by Soker \& Tylenda (2003). A more remarkable consequence is that this scenario will be able to explain the hydrogen-rich appearance (Naylor et al. 1992) of the proposed remnant object of CK Vul, which is unlike a hydrogen-poor Wolf-Rayet-like object (Clayton \& de Marco 1997), R CrB-like object, or cooling white dwarf what are expected from the final helium-flash scenario.

The nebula surrounding CK Vul (Shara \& Moffat 1982; Shara et al. 1985) is also unusual for a planetary nebula. Shara et al. (1985) suggested, from an analysis of emission lines and an estimate of the recombination time, that the nebula is excited by a shock which was produced by a collision of the CK Vul wind (or ejecta) with the interstellar matter. Such a feature has not been observed in final helium-flash objects, while an expected heavy mass-loss from a merging binary can more reasonably explain the origin. An estimated nebular expansion velocity of $59 \pm 16 \mathrm{~km} \mathrm{~s}^{-1}$ is reasonable compared to the outflows with the observed velocities of $200-500 \mathrm{~km} \mathrm{~s}^{-1}$ in V838 Mon considering an effect of deceleration. Such an outflow was not observed in the final helium-flash object, V4334 Sgr (Duerbeck et al. 1997).

Finally, we compare the outburst absolute magnitudes between CK Vul and V838 Mon. With a long-distance estimate $(\sim 3 \mathrm{kpc}, \mathrm{H} \text {. E. Bond, cited in Soker \& Tylenda 2003) })^{1}$, and a reasonable extinction of $A_{V} \sim 1.5$ (Munari et al. 2002b), the maximum $M_{V}$ of V838 Mon is estimated to be -7.0. This value is appreciably close to $M_{V} \sim-8$ by adopting the estimated distance of $550 \mathrm{pc}$ and of $A_{V} \sim 2.2$ in CK Vul (Shara et al. 1985). Shara et al. (1985) noted that some of Helvelius' 1670-1671 observations described CK Vul as "blurred". This

\footnotetext{
The estimated distance of V838 Mon significantly differs between the authors. For example, Munari et al. (2002b) suggested a distance of $790 \pm 30 \mathrm{pc}$ based on an assumption of the circumstellar origin of the light echo. The true distance is, however, still controversial (e.g. Munari et al. 2002a even discussed a possibility of $10.5 \mathrm{kpc}$ ). Since the variety of parameters in Soker \& Tylenda (2003) can naturally reproduce a diverse range of energetics, the ambiguity of the distance of V838 Mon would not be a serious problem in interpreting CK Vul itself, although the deduced similarity with V838 Mon might become less striking.
} 
intriguing finding may be somehow related to a V838 Mon-like light echo (Munari et al. 2002b), although it seems rather unlikely to resolve such a light echo with the unaided eyes even at a small distance. Considering the ambiguity of the distance estimates of these objects, the 1670 outburst of CK Vul may be best understood as a V838 Mon-like event which occurred in our vicinity. Future detailed observations of the CK Vul remnant in view of the present possibility could provide a crucial test for this interpretation.

Acknowledgements. We are grateful to the referee (A. Evans) for drawing the author's attention to the description of Helvelius' 1670-1671 observations of CK Vul. We are grateful to the observers who reported vital observations to VSNET (http://www.kusastro.kyoto-u.ac.jp/vsnet/). This work is partly supported by a grant-in-aid (13640239) from the Japanese Ministry of Education, Culture, Sports, Science and Technology.

\section{References}

Asplund, M., Lambert, D. L., Kipper, T., Pollacco, D., \& Shetrone, M. D. 1999, A\&A, 343, 507
Brown, N. J., Waagen, E. O., Scovil, C., et al. 2002, IAU Circ., 7785

Clayton, G. C., \& de Marco, O. 1997, AJ, 114, 2679

Duerbeck, H. W. 1981, PASP, 93, 165

Duerbeck, H. W. 1987, Space Sci. Rev., 45, 1

Duerbeck, H. W., Benetti, S., Gautschy, A., et al. 1997, AJ, 114, 1657

Evans, A., van Loon, J. T., Zijlstra, A. A., et al. 2002, MNRAS, 332, $35 \mathrm{P}$

Harrison, T. E. 1996, PASP, 108, 1112

Hjellming, R. M., \& Taam, R. E. 1991, ApJ, 370, 709

Kerber, F. 1999, New Astron. Rev., 43, 425

Munari, U., Desidera, S., \& Henden, A. 2002a, IAU Circ., 8005

Munari, U., Henden, A., Kiyota, S., et al. 2002b, A\&A, 389, L51

Naylor, T., Charles, P. A., Mukai, K., \& Evans, A. 1992, MNRAS, 258,449

Payne-Gaposchkin, C. 1957, The Galactic Novae (Amsterdam: North-Holland)

Prialnik, D., \& Livio, M. 1985, MNRAS, 216, 37

Shara, M. M., Livio, M., Moffat, A. F. J., \& Orio, M. 1986, ApJ, 311, 163

Shara, M. M., \& Moffat, A. F. J. 1982, ApJ, 258, L41

Shara, M. M., Moffat, A. F. J., \& Webbink, R. F. 1985, ApJ, 294, 271

Soker, N., \& Tylenda, R. 2003, ApJ, 582, L105 